\title{
New Perspective On Space And Time From Lorentz Violation*
}

\author{
BO-QIANG MA \\ School of Physics and State Key Laboratory of Nuclear Physics and Technology, Peking \\ University, Beijing 100871, China \\ Center for High Energy Physics, Peking University, Beijing 100871, China \\ Center for History and Philosophy of Science, Peking University, Beijing 100871, China \\ mabq@pku.edu.cn
}

\begin{abstract}
I present a brief review on space and time in different periods of physics, and then talk on the nature of space and time from physical arguments. I discuss the ways to test such a new perspective on space and time through searching for Lorentz violation in some physical processes. I also make an introduce to a newly proposed theory of Lorentz violation from basic considerations.
\end{abstract}

Keywords: space; time; Lorentz violation.

\section{Space and Time in Physics}

Space and time have been discussed in human history over thousands of years, and their nature still remains mysterious to human beings. There are many issues concerning the nature of space and time, such as

- Whether space and time are existences or concepts?

- Whether they are objective or subjective?

- Whether they are continuous or discrete?

There are many speculations on these questions from different perspectives, such as from metaphysics, art, philosophy, or science. The speculations are also different in different periods of human history.

In physics, space and time are where all physical events take place. However, there are different understandings concerning the properties of space and time in different periods of physics.

In classical mechanics, space is 3 -dimensional and time is 1-dimensional, and they have the following properties

- Space and time are independent from each other;

*Plenary talk at First LeCosPA Symposium: Towards Ultimate Understanding of the Universe (LeCosPA2012), National Taiwan University, Taipei, Taiwan, February 6-9, 2012. 
- Space and time are objective and continuous;

- Time is universal and observer independent;

- Space are observer dependent.

The space and time provide the $3+1=4$ dimensions of degrees of freedom where the Newton's laws of motion and gravity act on objects existing in space and time. The space between different inertial frames of reference are connected by the Galileo transformation.

In 1905, Einstein established his theory of special relativity. The special relativity offers a revolution to the concepts of space and time in Newton's mechanics, and provides a theoretical derivation of adopting the Lorentz transformation for the covariance of the equations of electrodynamics, to replace the traditional Galileo transformation in classical physics. Space and time are unified into 4-dimensional space-time. The space and time in special relativity have the following properties

- Space and time are dependent with each other;

- Both space and time are observer dependent;

- Space and time are continuous and flat.

There are two basic principles of special relativity:

- Principle of Relativity: the equations describing the laws of physics have the same form in all inertial frames of reference.

- Principle of constant light speed: the speed of light is the same in all directions in vacuum in all reference frames, regardless whether the source of the light is moving or not.

These two principles lead to the unification of space and time into a 4-dimensional space-time satisfying the Lorentz symmetry.

To unify the relativity with Newton's law of gravity, Einstein developed his theory of general relativity during 1907-1915. Then the curvature of space-time is determined by energy and momentum distribution. The space and time in general relativity have the following properties

- Space and time are dependent with each other;

- Both space and time are observer and also matter-distribution dependent;

- Space and time are continuous and can be curved.

One of the essence of general relativity is the principle of equivalence between gravity and inertial force, and this means that every observer can find a local inertial frame which is free from any gravitational effect. Thus the Lorentz symmetry always holds in such kind of local inertial frames.

Einstein's theories of relativity have been proved to be valid at very high precision and thus have achieved great triumphs. The Lorentz invariance, i.e., that statement that physical laws keep invariant under the Lorentz transformation, becomes a basic theoretical foundation of physics. Then we need to face the question: 
- Is there any reason that we seek for Lorentz violation?

The Lorentz symmetry is a symmetry related with space and time, therefore the Lorentz violation should be related to the basic understandings of space and time. From the viewpoint of physics, the origin for the breaking down of conventional concepts of space and time might be traced back to Planck. With three fundamental constants in physics: the Newton gravitational constant $G$, the light speed in vacuum $c$, and the Boltzmann constant $k_{B}$, Planck introduced a new constant $\hbar$ In 1899, for the purpose to construct a "God-given" unit system. ${ }^{1}$ By setting the above four constants as bases, one can construct the Planck unit system with a number of basic quantities, such as the Planck length $l_{\mathrm{P}} \equiv \sqrt{G \hbar / c^{3}} \simeq 1.6 \times 10^{-35} \mathrm{~m}$, the Planck time $t_{\mathrm{P}} \equiv \sqrt{G \hbar / c^{5}} \simeq 5.4 \times 10^{-44} \mathrm{~s}$, the Planck energy $E_{\mathrm{P}} \equiv \sqrt{\hbar c^{5} / G} \simeq 2.0 \times 10^{9} \mathrm{~J}$, and the Planck temperature $T_{\mathrm{P}} \equiv \sqrt{\hbar c^{5} / G k_{B}^{2}} \simeq 1.4 \times 10^{32} \mathrm{~K}$. Therefore one may suspect that conventional understanding of space and time might be breaking down at the Planck scale: ${ }^{2}$ i.e., at the Planck length $l_{\mathrm{P}}$, or the Planck time $t_{\mathrm{P}}$, or the Planck energy $E_{\mathrm{P}}$, where new features of existence may emerge. The breaking down of continue space-time was also conjectured. ${ }^{3,4}$ The expectation for the existence of a minimal length as the Planck length led also to the establishment of some theories, e.g., the doubly special relativity (DSR). ${ }^{5,6}$

Just recently, $\mathrm{Xu}$ and I provided a physical argument for the discreteness of space and time. ${ }^{7}$ From two known entropy constraints:

$$
S_{\text {matter }} \leq 2 \pi E R, \quad \text { and } S_{\text {matter }} \leq \frac{A}{4},
$$

combined with the black-body entropy,

$$
S=\frac{4}{45} \pi^{2} T^{3} V=\left(\frac{16}{135}\right) \pi^{3} R^{3} T^{3}
$$

we arrive at a minimum value of space

$$
R \geq\left(\frac{128}{3645 \pi}\right)^{\frac{1}{2}} l_{\mathrm{P}} \simeq 0.1 l_{\mathrm{P}}
$$

Thus we reveal from physical arguments that space-time is discrete rather than continuous. From another point of view, the newly proposed entropic gravity suggests gravity as an emergent force rather than a fundamental one. ${ }^{8,9}$ If gravity is emergent, a new fundamental constant should be introduced to replace the Newtonian constant $G .^{2}$ It is natural to suggest a fundamental length scale, and such constant can be explained as the smallest length scale of quantum space-time. Its value can be measured through searches of Lorentz violation. ${ }^{2,7}$ The existence of an "æether" (or a "vacuum" at rest in a specific frame) can also bring the breaking down of Lorentz invariance. ${ }^{10,11}$

Therefore the research on the Lorentz violation may provide us the chance for new understanding of the nature of basic concepts, such as "space", "time", and "vacuum", through physical ways, rather than from the perspectives of metaphysics or philosophy. It is thus necessary to push forward the studies on Lorentz violation from both theoretical and experimental aspects. 


\section{A Glimpse on Lorentz Violation Studies}

Nowadays, there has been an increasing interest in Lorentz invariance Violation (LV or LIV) both theoretically and experimentally. The possible Lorentz symmetry violation effects have been sought for from various theories, motivated by the unknown underlying theory of quantum gravity together with various phenomenological applications. ${ }^{12-17}$ This can happen in many alternative theories, e.g., the doubly special relativity (DSR), ${ }^{5,6,18}$ torsion in general relativity, ${ }^{19-21}$ and large extra-dimensions ${ }^{22,23}$ et al.. As examples, I list below some phenomenological consequences of the Lorentz violation effects studied by my students and I in the last few years:

- The Lorentz violation could provide an explanation of neutrino oscillation without neutrino mass. ${ }^{24,25}$ We carried out Lorentz violation contribution to neutrino oscillation by the effective field theory for Lorentz violation and give out the equations of neutrino oscillation probabilities. In our model, neutrino oscillations do not have drastic oscillation at low energy and oscillations still exist at high energy. It is possible that neutrinos may have small mass and both Lorentz violation and the conventional oscillation mechanisms contribute to neutrino oscillation.

- The modified dispersion relation of the proton could increase the threshold energy of photo-induced meson production of the proton and cause an increase of the GZK cutoff energy. The earlier reports on super-GZK events triggered attention on Lorentz-Violation. The new results of observation of GZK cut-off put strong constraints on Lorentz violation parameters. ${ }^{24}$

- The modified dispersion relation of the photon may cause time lag of photons with different energies when they propagate in space from far-away astro-objects. The Lorentz violation can modify the photon dispersion relation, and consequently the speed of light becomes energy-dependent. ${ }^{13}$ This results in a tiny time delay between high energy photons and low energy ones. Very high energy photon emissions from cosmological distance can amplify these tiny LV effects into observable quantities. We analyzed photons from $\gamma$-ray bursts from Fermi satellite observations and presented a first robust analysis of these taking the intrinsic time lag caused by sources into account, and gave an estimate to LV energy scale $\sim 2 \times 10^{17} \mathrm{GeV}$ for linear energy dependence, and $\sim 5 \times 10^{9} \mathrm{GeV}$ for quadratic dependence. ${ }^{14}$

- We also studied recent data on Lorentz violation induced vacuum birefringence from astrophysical consequences. ${ }^{15}$ Due to the Lorentz violation, two helicities of a photon have different phase velocities and group velocities, termed as "vacuum birefringence". From recently observed $\gamma$-ray polarization from Cygnus X-1, we obtained an upper limit $\sim 8.7 \times 10^{-12}$ for Lorentz-violating parameter $\chi$, which is the most firm constraint from well-known systems. 


\section{A Newly Proposed Theory of Lorentz Violation from Basic Principles}

Among many theoretical investigations of Lorentz violation, it is a powerful framework to discuss various LV effects based on traditional techniques of effective field theory in particle physics. Here we focus our attention on a newly proposed theory of Lorentz violation from basic principles: the Standard Model Supplement (SMS). ${ }^{26,27}$

It is clear that human should not be narrowly focused just on effective theories with some additional terms beyond the conventional theory added by hand. It is a basic requirement that we should find a fundamental theory to derive the Lorentz violation terms from basic consideration. In the standard model supplement (SMS) framework, ${ }^{26,27}$ the LV terms are brought about from a basic principle denoted as the physical independence or physical invariance (PI):

- Principle of Physical Invariance: the equations describing the laws of physics have the same form in all admissible mathematical manifolds.

The principle leads to the following replacement of the ordinary partial $\partial_{\alpha}$ and the covariant derivative $D_{\alpha}$

$$
\partial^{\alpha} \rightarrow M^{\alpha \beta} \partial_{\beta}, \quad D^{\alpha} \rightarrow M^{\alpha \beta} D_{\beta},
$$

where $M^{\alpha \beta}$ is a local matrix. The Lorentz violation terms are thus uniquely determined from the standard model Lagrangian without any ambiguity, ${ }^{26}$ and their general existence is derived from basic consideration rather than added by hand. The explicit form of the matrices $M^{\alpha \beta}$ demands more basic theories concerning the true nature of space and time, and we suggest to adopt a physical way to explore these matrices through experiments rather than from theory at first. For more generality, we do not make any ad hoc assumption about these matrices. Thus these matrices might be particle dependent corresponding to the standard model particles under consideration, with the elements of these matrices to be measured or constrained from experimental observations.

We separate $M^{\alpha \beta}$ to two matrices like $M^{\alpha \beta}=g^{\alpha \beta}+\Delta^{\alpha \beta}$, where $g^{\alpha \beta}$ is the metric tensor of space-time and $\Delta^{\alpha \beta}$ is a new matrix which is particle-type dependent generally. Since $g^{\alpha \beta}$ is Lorentz invariant, $\Delta^{\alpha \beta}$ contains all the Lorentz violating degrees of freedom from $M^{\alpha \beta}$. Then $\Delta^{\alpha \beta}$ brings new terms violating Lorentz invariance in the standard model and is called Lorentz violation matrix. The theory returns back to the standard model when these Lorentz violation matrices vanish.

More explicitly, the effective Lagrangian $\mathcal{L}_{\mathrm{SM}}$ of the minimal standard model is composed of four parts

$$
\begin{aligned}
\mathcal{L}_{\mathrm{SM}} & =\mathcal{L}_{\mathrm{G}}+\mathcal{L}_{\mathrm{F}}+\mathcal{L}_{\mathrm{H}}+\mathcal{L}_{\mathrm{HF}}, \\
\mathcal{L}_{\mathrm{G}} & =-\frac{1}{4} F^{a \alpha \beta} F_{\alpha \beta}^{a}, \\
\mathcal{L}_{\mathrm{F}} & =i \bar{\psi} \gamma^{\alpha} D_{\alpha} \psi, \\
\mathcal{L}_{\mathrm{H}} & =\left(D^{\alpha} \phi\right)^{\dagger} D_{\alpha} \phi+V(\phi),
\end{aligned}
$$


where we omit the chiral differences, the summation of chirality and gauge scripts. $\psi$ is the fermion field, $\phi$ is the Higgs field, and $V(\phi)$ is the Higgs self-interaction. $F_{\alpha \beta}^{a}=\partial_{\alpha} A_{\beta}^{a}-\partial_{\beta} A_{\alpha}^{a}-g f^{a b c} A_{\alpha}^{b} A_{\beta}^{c}, D_{\alpha}=\partial_{\alpha}+i g A_{\alpha}$ and $A_{\alpha}=A_{\alpha}^{a} t^{a}$, with $A_{\alpha}^{a}$ being the gauge field. $g$ is the coupling constant, and $f^{a b c}$ and $t^{a}$ are the structure constants and generators of the corresponding gauge group respectively. $\mathcal{L}_{\mathrm{HF}}$ is the Yukawa coupling between the fermions and the Higgs field, and is not related to derivatives, thus it remains unchanged under the replacement (44).

Under (44) and the decomposition $M^{\alpha \beta}=g^{\alpha \beta}+\Delta^{\alpha \beta}$, the Lagrangians in (6)-(8) become

$$
\begin{aligned}
\mathcal{L}_{\mathrm{G}} & =-\frac{1}{4}\left(M^{\alpha \mu} \partial_{\mu} A^{a \beta}-M^{\beta \mu} \partial_{\mu} A^{a \alpha}-g f^{a b c} A^{b \alpha} A^{c \beta}\right) \\
& \times\left(M_{\alpha \mu} \partial^{\mu} A_{\beta}^{a}-M_{\beta \mu} \partial^{\mu} A_{\alpha}^{a}-g f^{a b c} A_{\alpha}^{b} A_{\beta}^{c}\right) \\
& =-\frac{1}{4} F^{a \alpha \beta} F_{\alpha \beta}^{a}+\mathcal{L}_{\mathrm{GV}}, \\
\mathcal{L}_{\mathrm{F}} & =i \bar{\psi} \gamma_{\alpha} M^{\alpha \beta} D_{\beta} \psi=i \bar{\psi} \gamma^{\alpha} D_{\alpha} \psi+\mathcal{L}_{\mathrm{FV}}, \\
\mathcal{L}_{\mathrm{H}} & =\left(M^{\alpha \mu} D_{\mu} \phi\right)^{\dagger} M_{\alpha \nu} D^{\nu} \phi+V(\phi) \\
& =\left(D^{\alpha} \phi\right)^{\dagger} D_{\alpha} \phi+V(\phi)+\mathcal{L}_{\mathrm{HV}},
\end{aligned}
$$

with $M^{\alpha \beta}$ being the real matrix to maintain the Lagrangian hermitian. The last three terms $\mathcal{L}_{\mathrm{GV}}, \mathcal{L}_{\mathrm{FV}}$ and $\mathcal{L}_{\mathrm{HV}}$ of the equations mentioned above are the supplementary terms for the ordinary Standard Model. The explicit forms of these terms are

$$
\begin{aligned}
\mathcal{L}_{\mathrm{GV}}= & -\frac{1}{2} \Delta^{\alpha \beta} \Delta^{\mu \nu}\left(g_{\alpha \mu} \partial_{\beta} A^{a \rho} \partial_{\nu} A_{\rho}^{a}-\partial_{\beta} A_{\mu}^{a} \partial_{\nu} A_{\alpha}^{a}\right) \\
& -F_{\mu \nu}^{a} \Delta^{\mu \alpha} \partial_{\alpha} A^{a \nu} \\
\mathcal{L}_{\mathrm{FV}}= & i \Delta^{\alpha \beta} \bar{\psi} \gamma_{\alpha} \partial_{\beta} \psi-g \Delta^{\alpha \beta} \bar{\psi} \gamma_{\alpha} A_{\beta} \psi, \\
\mathcal{L}_{\mathrm{HV}}= & \left(g_{\alpha \mu} \Delta^{\alpha \beta} \Delta^{\mu \nu}+\Delta^{\beta \nu}+\Delta^{\nu \beta}\right)\left(D_{\beta} \phi\right)^{\dagger} D_{\nu} \phi .
\end{aligned}
$$

Thus we obtain a new effective Lagrangian for the Standard Model with new supplementary terms, denoted by $\mathcal{L}_{\mathrm{SMS}}$

$$
\begin{aligned}
\mathcal{L}_{\mathrm{SMS}} & =\mathcal{L}_{\mathrm{SM}}+\mathcal{L}_{\mathrm{LV}}, \\
\mathcal{L}_{\mathrm{LV}} & =\mathcal{L}_{\mathrm{GV}}+\mathcal{L}_{\mathrm{FV}}+\mathcal{L}_{\mathrm{HV}},
\end{aligned}
$$

where $\mathcal{L}_{\text {SMS }}$ satisfies the Lorentz covariance $\left(\mathrm{SO}^{+}(1,3)\right)$, the gauge symmetry invariance of $\mathrm{SU}(3) \times \mathrm{SU}(2) \times \mathrm{U}(1)$ and invariance under the requirement of the principle of physical invariance or independence $(\mathrm{PI})$, under which $\mathcal{L}_{\mathrm{SM}}$ cannot remain unchanged in a general situation.

We can have a better understanding of the LV terms in SMS here. The elements of $M^{\alpha \beta}$ of a particle are mass dimensionless (which is natural for the sign of testifying the Lorentz invariance), and they are not global constants generally. All of the LV terms are expressed in $\mathcal{L}_{\mathrm{LV}}$, and the $\mathrm{LV}$ information is measured by the concise matrix $\Delta^{\alpha \beta}$, which is convenient for a systematic study of the LV effects. To determine whether the Lorentz invariance holds exactly, further work 
is needed to analyze the effective Lagrangian (15) of QED, QCD and EW (ElectroWeak) fields, and more experiments are needed to determine the magnitude of the elements in the matrices $M^{\alpha \beta}$ for different particles. There have been some preliminary progress along this line with the theory of SMS applied to discuss the Lorentz violation effects for protons,${ }^{26}$ photons, ${ }^{27}$ and neutrinos. ${ }^{28}$ More works are still needed for systematic studies.

Generally, $\Delta^{\alpha \beta}$ might be particle-type and flavor-type dependent. If we use the vacuum expectation values of a $\Delta^{\alpha \beta}$ for the coupling constants in the corresponding effective Lagrangian, not all of the 16 degrees of freedom of $M^{\alpha \beta}$ are physical. For the derivative field $M\left(\partial_{x}\right) \varphi(x)$ of an arbitrary given field, $\varphi(x)$ can be rescaled to absorb one of the 16 degrees of freedom so that only 15 are left. When more fields are involved, there is only one degree of freedom that can be reduced from a rescaling consideration for all fields. Thus for generality, we may keep all 16 degrees of freedom in $M^{\alpha \beta}$ for a specific particle in our study.

The matrix $M^{\alpha \beta}$ in the SMS theory just appears with the derivative terms of Lagrangians, but not with the coordinate terms. Therefore one should not confuse this matrix with the metric of the general relativity. To define a covariant derivative in general relativity, one needs to introduce the concept of connection to reflect the effect due to the curvature of space and time from gravity. Our introduction of the matrix $M^{\alpha \beta}$ can be considered as an alternative choice along the similar philosophy, but with a more general sense by including also possible effects from other interactions other than solely gravity. Therefore our matrix $M^{\alpha \beta}$ can contain the effect due to general relativity or more beyond that, but we do not intend to derive it from theory but to detect it from phenomenological manifestations. This is from the consideration that the space-time structure of nature might be more complicated than just the effect from gravity.

There still exists the question of how to understand and handle the Lorentz violation matrix $\Delta^{\alpha \beta}$. We list here three options for understandings and treatments: ${ }^{29}$

- Scenario I: which can be called as fixed scenario in which the Lorentz violation matrices are taken as constant matrices in any inertial frame of reference the observer is working. It means that the Lorentz violation matrices can be taken as approximately the same for any working reference frames such as the earth-rest frame, the sun-rest frame, or the CMB frame. However, there will be the problem of inconsistency for an "absolute physical event" between different reference frames, ${ }^{30}$ if one sticks to this scenario. Therefore this scenario can be adopted as a practical approach when one is focused on the Lorentz violation effect within a certain frame and does not care about relationships between different frames.

- Scenario II: which can be called as "new æether" scenario in which the Lorentz violation matrices transform as tensors between different inertial frames but keep as constant matrices within the same frame. The Lorentz violation matrices play the roles for the exitance of some kinds of back- 
ground fields, or the "new æether" (i.e., a "vacuum" at rest in a specific frame), which changes from one frame to another frame by Lorentz transformation. Thus it can be considered as a standard viewpoint to treat the matrix $M^{\alpha \beta}$ as a tensor satisfying Lorentz symmetry.

- Scenario III: which can be called as covariant scenario in which the Lorentz violation matrices transform as tensors adhered with the corresponding standard model particles. It means that these Lorentz violation matrices are emergent and covariant with their standard model particles. Such a scenario still needs to be checked for consistency and for applications in future.

Before accepting the SMS as a fundamental theory, one can take the SMS as an effective framework for phenomenological applications by confronting with various experiments to determine and/or constrain the Lorentz violation matrioce $\Delta^{\alpha \beta}$ for various particles. So our idea is to reveal the real structure of Lorentz violation of nature from experiments rather than from theory. We consider this phenomenological way as more appropriate for physical investigations, rather than to derive everything from theory at first. As a comparison, the specific form of the quark mixing matrix is determined from experimental measurements rather than derived from theory. ${ }^{31}$ Even after so many years of research and also the elements of the CKM mixing matrix have been measured to very high precision, there is still no a commonly accepted theory to derive the quark mixing matrix from basic principles.

We now provide some remarks concerning the Lorentz violation studies in field theory frameworks. In the effective field theory frameworks, the standard particles transform according to the Lorentz symmetry between different momentum states. The background fields should also transform according to the Lorentz symmetry between different observer working frames from the requirement of consistency. From this sense, there is actually no Lorentz violation for the whole system of the standard model particles together with the background fields. The Lorentz violation exists for the standard model particles within an observer working frame, when these particles have different momenta between each other. From this sense, the Lorentz violation is due to the existence of the background fields, which are treated as fixed parameters in the observer working frame.

The newly proposed theory of SMS not only provides clear relationship between some general LV parameters, ${ }^{32}$ but also can be conveniently applied for phenomenological analysis. ${ }^{33}$ We would need more experimental investigations to check whether it can meet the criterion of being able to provide a satisfactory description of the physical reality, with simplicity and beauty in formalism, together with the predictive power towards new knowledge for human beings. It is also possible that the nature satisfies the Lorentz symmetry perfectly and we would be unable to find a physical evidence to support the theory. This would imply that the newly introduced Lorentz violation matrix $\Delta^{\alpha \beta}$ would vanish for nature. 


\section{Conclusion}

From the discussions above, we may have some new perspectives on the nature of space-time:

- Space and time are $3+1=4$ dimensional or may have extra-dimensions;

- Both space and time are observer and object dependent;

- Space and time might be particle-type dependent and can be curved by the existence of matter.

- Space and time might be discrete and such discreteness can be tested through experiments on Lorentz violation.

Finally we present our conclusion:

- Researches on Lorentz violation have been active for many years;

- There might be some marginal evidences for Lorentz violation yet, but non of them can be considered as confirmed;

- The Lorentz violation study can bring conceptual revolution on the understanding of space-time for human beings;

- Lorentz violation is being an active frontier both theoretically and experimentally.

\section{Acknowledgements}

I acknowledge Professor Pisin Chen for his warm invitation and hospitality for attending the 1st LeCosPA Symposium. I am very grateful for the discussions and collaborations with a number of my students: Zhi Xiao, Shi-Min Yang, Lijing Shao, Lingli Zhou, Xinyu Zhang, Yunqi Xu, and Nan Qin, who devoted their wisdoms and enthusiasms bravely on the topic of Lorentz violation in past few years. The work was supported by National Natural Science Foundation of China (Nos. 10975003, 11021092, 11035003 and 11120101004).

\section{References}

1. M. Planck, Sitzber. K. Preuss Aka. Berlin 5, 440 (1899).

2. L. Shao, B.-Q. Ma, Sci. China Phys. Mech. Astro. 54, 1771 (2011) arXiv:1006.3031 [hep-th]].

3. H.S. Snyder, Phys. Rev. 71, 38 (1947); 72, 68 (1947).

4. J.A. Wheeler, Ann. Phys. 2, 604 (1957).

5. G. Amelino-Camelia, Int. J. Mod. Phys. D 11, 35 (2002) arXiv:gr-qc/0012051.

6. J. Magueijo and L. Smolin, Phys. Rev. Lett. 88, 190403 (2002) arXiv:hep-th/0112090].

7. Y. Xu and B.-Q. Ma, Mod. Phys. Lett. A 26, 2101 (2011) arXiv:1106.1778 [hep-th]].

8. E. P. Verlinde, JHEP 1104, 029 (2011) arXiv:1001.0785 [hep-th]].

9. X. G. He and B.-Q. Ma, Chin. Phys. Lett. 27, 070402 (2010) arXiv:1003.1625 [hep-th]].

10. P.A.M. Dirac, Nature 168, 906 (1951).

11. J.D. Bjorken, Ann. Phys. 24, 174 (1963).

12. For a brief review on Lorentz violation effects through very high energy photons of 
astrophysical sources, see. e.g., L. Shao, B.-Q. Ma, Mod. Phys. Lett. A 25, 3251 (2010) arXiv:1007.2269, and references therein.

13. Z. Xiao, B.-Q. Ma, Phys. Rev. D 80, 116005 (2009) arXiv:0909.4927 [hep-ph]].

14. L. Shao, Z. Xiao, B.-Q. Ma, Astropart. Phys. 33, 312 (2010) arXiv:0911.2276 [hep$\mathrm{ph}]$.

15. L. Shao and B. -Q. Ma, Phys. Rev. D 83, 127702 (2011) arXiv:1104.4438 [astroph.HE]].

16. Z. Xiao, L. Shao, B.-Q. Ma, Eur. Phys. J. C 70, 1153 (2010) arXiv:1011.5074 [hepth]].

17. W. Bietenholz, Phys. Rept. 505, 145 (2011).

18. X. Zhang, L. Shao, B.-Q. Ma, Astropart. Phys. 34, 840 (2011) arXiv:1102.2613 [hepth]].

19. W.-T. Ni, Phys. Rev. Lett. 35, 319 (1975).

20. W.-T. Ni, Rept. Prog. Phys. 73, 056901 (2010) arXiv:0912.5057 [gr-qc]].

21. M.L. Yan, Commun. Theor. Phys. 2, 1281 (1983).

22. V. Ammosov and G. Volkov, hep-ph/0008032

23. H. Pas, S. Pakvasa, T.J. Weiler, Phys. Rev. D 72, 095017 (2005).

24. Z. Xiao, B.-Q. Ma, Int. J. Mod. Phys. A 24, 1359 (2009) arXiv:0805.2012.

25. S. Yang, B.-Q. Ma, Int. J. Mod. Phys. A 24, 5861 (2009) arXiv:0910.0897.

26. Zhou L., B.-Q. Ma, Mod. Phys. Lett. A 25, 2489 (2010) arXiv:1009.1331.

27. Zhou L., B.-Q. Ma, Chin. Phys. C 35, 987 (2011) arXiv:1109.6387.

28. Zhou L., B.-Q. Ma, arXiv:1109.6097.

29. B.-Q. Ma, Mod. Phys. Lett. A 27, 1230005 (2012) arXiv:1111.7050 [hep-ph]].

30. B. Q. Ma, Int. J. Mod. Phys. Conf. Ser. (2012) in press arXiv:1203.0086 [hep-ph]].

31. B.-Q. Ma, Int. J. Mod. Phys. Conf. Ser. 1, 291 (2011) arXiv:1109.5276 [hep-ph]].

32. Zhou L., B.-Q. Ma, arXiv:1110.1850 [hep-ph].

33. Zhou L., B.-Q. Ma, arXiv:1009.1675. 